Dhaka Univ. J. Sci. 60(2): 169-173, 2012 (July)

\title{
Quality of Industrially Processed Fruit Juices: An Assessment Using Multivariate Framework
}

\author{
Md. Anwar Hossain ${ }^{1}$, Matiur Rahman² and Md. Zillur Rahman Shabuz ${ }^{2}$ \\ ${ }^{1}$ Bangladesh Council of Scientific and Industrial Research, Dhaka-1205, Bangladesh \\ ${ }^{2}$ Department of Statistics, Biostatistics and Informatics, University of Dhaka, Dhaka-1000, Bangladesh
}

Received on 14. 05. 2011. Accepted for Publication on 22. 02. 2012.

\begin{abstract}
A multivariate statistical technique, called Discriminant Analysis (DA) has been applied for the evaluation of fruit juices quality variations and the interpretation of juices quality. Data set on two types namely, mango (Mangifera indica) and orange (Citrus sinensis) fruit juices in Bangladesh incorporating 17 variables for 9 different local manufacturing companies have been used. One sample $t$-test and independent samples $t$-test were also used to analyze the physico-chemical values and microbiological counts. DA gave the best result to identify the relative contribution of variables in discriminating (distinguishing) the two types of juices affording $100 \%$ correct assignations. This study illustrates the benefit of multivariate statistical techniques for analyzing and interpretation of complex data sets and to plan for future studies. DA indicated that copper was the main contributor in discriminating the two types of fruit juices. The $t$-tests results obtained showed that there were no significant differences in acidity, arsenic, lead, zinc, copper and standard plate count content between samples standards, while significant differences were observed in total soluble solid, ash and vitamin $\mathrm{C}$ compositions between samples standards.
\end{abstract}

Keywords: Discriminant Analysis (DA), mango (Mangifera indica), orange (Citrus sinensis) and $t$-tests.

\section{Introduction}

Fruit juices are becoming an important part of the modern diet in many communities. It acts as a nutritious beverage and can play a significant part in a healthy diet because they offer good taste and a variety of nutrients found naturally in fruits. Juices are available in their natural concentrations or in processed forms.

Juice is prepared by mechanically squeezing fresh fruits or may be extracted by water. Juices are fat-free, nutrientdense beverages that are rich in vitamins, minerals and naturally occurring phytonutrients that contribute to good health. For example, orange juice is rich in vitamin $\mathrm{C}$, an excellent source of bio-available antioxidant phytochemicals (Franke, et al., 2005) and significantly improves blood lipid profiles in people affected with hypercholesterolemia (Kurowska, et al. 2000). Fruit juices promote detoxification in the human body (Deanna and Jeffrey, 2007).

The ingredients of processed juices contain mainly water, sugar, preservatives, colour and fruits pulps. The most commonly used preservatives are benzoic acid, sorbic acid or sulphur dioxide. Natural colours such as anthocynins and betanin are used. Acid is an essential universal constituent of Juice and the most acid commonly used is citric acid.

Heavy metals occur in all foods as natural or inherent components of plant and animal tissues and fluid and also may be present as a result of contamination or deliberate addition (Underwood, 1973). One form of environmental contamination arises from exposure to water polluted by industrial waste (Mathew, 1986).

Most fruit juices contain sufficient nutrients that could support microbial growth. Several factors encourage, prevent or limit the growth of microorganisms in juices; the most important are $\mathrm{pH}$, hygienic practice storage temperature and concentration of preservatives. Storage of products at refrigeration temperature or below is not always best for the maintenance of desirable quality of some fruits. Water used for juice preparation can be a major source of microbial contaminants such as total coliforms, faecal coliforms, faecal streptococci, etc. Environmental fomites may also make the fruits unsafe and these may have a role in spreading of Salmonella, Shigella, Vibrio, Escherichia coli, and other diseases causing as well as fruits spoilage types (Doyle, Beuchat \& Montville, 2001.). Spoilage yeasts, such as Saccharomyces cerevisiae, Candida lipolytica and Zygosaccharomyces spp. can tolerate acidic environments. It should also be noted that changes in $\mathrm{pH}$ could transform a food into one, which can support growth of pathogens (FDA, 2001).

The quality of fruit juices is strictly maintained in developed countries under some law and regulation but in many developing and under developed countries the manufacturer is not concerned about the microbiological safety and hygiene of fruit juices because of negligence of law. Thus the transmission of some human diseases through juice and other drinks are considered a serious problem in recent years.

Fruit juices are available in essentially the same form almost anywhere in the world. From polar bases to the tropics and from the largest developed countries, fruit juices are available in bottles, cans, laminated paper packs, pouches, cups and almost every other form of packaging known. In recent years these juices have been included significantly in diet of every person irrespective to age. So maintaining the quality of processed fruit juices is important issue now. In order to develop awareness among the people about fruit juices in transmitting diseases this study was attempted to measure nutritional and microbiological quality of industrially processed locally available fruit juices. 
The application of multivariate methods such as Cluster Analysis (CA), Principal Component Analysis (PCA), Factor Analysis (FA) and Discriminant Analysis (DA) has increased tremendously in recent years for analyzing environmental data and drawing meaningful information (Vega et al., 1998; Lee et al., 2001; Saadia, et al., 2005). In this paper we report our findings of the study of fruit juice quality of two types such as mango (mangifera indica) and orange (citrus sinensis) juices in Bangladesh and their statistical analysis.

\section{Data}

Seven types of mango (mangifera indica) juices and two types of orange (citrus sinensis) juices were collected from different prevented manufacturer (Acme, Pran, Starship, Danish, Shezan and Akiz Group) in the Dhaka City, for nutritional and microbiological analysis. Samples were collected from March 2009 through December 2009. At least seven samples of each category were analyzed to overcome the sampling biasness. These samples are commercial name as Acme orange, Acme mango, Pran mango, Starship mango, Shezan mango, Acme premium orange, Frooto mango, Frutika mango and Danish mango.

\section{Variables}

1. Physico-chemical variables such as moisture, ash, crude protein, ascorbic acid, total sugar, reducing sugar, total soluble solid (TSS), acidity and $\mathrm{pH}$.

2. Microbiological variables are Standard plate count, Total Coliform, Total Fungus and Salmonella.

3. Heavy metals variables are Arsenic, Lead, Zinc and Copper.

These are shortly discussed below:

\section{Estimation of nutrient composition of fruit juices}

Moisture and ash contents of fruit juices were determined using standard AOAC methods (Horwitz W, 2003). Crude Protein content of the samples was determined using the Kjeldahl method (AOAC) (Horwitz W, 2003). The method consists of three basic steps: (i) digestion of the sample in sulfuric acid with a catalyst, which results in conversion of nitrogen to ammonia; (ii) distillation of the ammonia into a trapping solution; and (iii) quantification of the ammonia by titration with a standard solution. According to this method, $\%$ crude protein content of a sample $=\%$ nitrogen $\times 6.25$.

Ascorbic acid was estimated by 2, 6-Dichlorophenolindophenol visual titration method according to AOAC. The reagents used for the estimation of vitamin-C were as follows: 1) Metaphosphoric acid (6\%) 2) Standard ascorbic acid solution 3) 2, 6-Dichlorophenolindophenol dye. For estimation of vitamin-C, the following steps were followed: standardization of dye solution, preparation of solution and titration (AOAC, 2004). The official Lane- Eynon method described in AOAC was used to measure the sugar contents (total sugar and reducing sugar) in fruit juices (James, 2004).

Total Soluble Solid (TSS) is one of the most important quality factors for most of fruit juices. TSS content of fruit juices was determined by using an Abbe refractrometer by placing a drop of pulp solution on its prism. The percentage of TSS was obtained from direct reading of the refractrometer.

Acidity was determined by dissolving a known weight of sample in distilled water and titration against $0.01 \mathrm{~N} \mathrm{NaOH}$ using phenolphthalein as indicator (Srivastava and Sanjeev, 2003) and Inolab digital $\mathrm{pH}$ meter was used for $\mathrm{pH}$ determination.

\section{Bacteriological analysis of the collected juice samples}

For the quantitative determination of total count of mesophilic bacteria, total coliform, faecal coliform, the standard procedure was followed (FDA, 2001). Standard plate count (SPC) was performed by pour plate method using plate count agar (PCA), which was incubated at $35 \pm 1^{0} \mathrm{C}$ for $48 \pm 2 \mathrm{~h}$. Lauryl tryptose broth was used for isolation of Escherichia coli. Gassing tube was selected for E.coli enumeration using most probable number (MPN) method. Enumeration of Fungi was performed on Potato Dextrose Agar medium. For the isolation of Salmonella species pre-enrichment was done by lactose broth followed by selective enrichment and finally it was confirmed using standard method (FDA, 2001).

\section{Heavy Metals}

Metals in fruit juices were measured using a graphite furnace of Atomic Absorption Spectrophotometer (GBC scientific equipment XAA1175, Australia) equipped with D2 background correction devices.

\section{Materials and Methods}

In this section we briefly highlight the analysis tools used for the study along with the rationale behind them.

Discriminant analysis (DA) is a multivariate technique used for two purposes, the first purpose is description of group separation in which linear functions of the several variables (discriminant functions (DFs)) are used to describe or elucidate the differences between two or more groups and identifying the relative contribution of all variable to separation of the groups. Second aspect is prediction or allocation of observations to group in which linear or quadratic functions of the variable (classification functions (CFs)) are used to assign an observation to one of the groups (Johnson \& Wichern, 2007).

Discriminant function analysis is used to determine which continuous variables discriminate between two or more naturally occurring groups. For example, a researcher may want to investigate which variables discriminate between fruits eaten by (1) primates, (2) birds, or (3) squirrels. For that 
purpose, the researcher could collect data on numerous fruit characteristics of those species eaten by each of the animal groups. Most fruits will naturally fall into one of the three categories. Discriminant analysis could then be used to determine which variables are the best predictors of whether a fruit will be eaten by birds, primates, or squirrels (Tabachnick and Fidell, 1996).

DA involves the determination of a linear equation like regression that will predict which group the case belongs to. The form of the equation or function is:

$$
D=v_{1} X_{1}+v_{2} X_{2}+\ldots \ldots \ldots+v_{i} X_{i}+a
$$

Where $D=$ discriminate function

$v=$ the discriminant coefficient or weight for that variable

$\mathrm{X}=$ respondent's score for that variable

$a=\mathrm{a}$ constant

$i=$ the number of predictor variables

For the present study DA can be used in order to identify differences between two groups such as Mango and Orange fruit juices.

\section{Why Use the t-test?}

Researchers use the t-test to compare two samples so that they can make an inference about the populations from which they drew the samples. Specifically, the t-test assesses whether the means of two groups are statistically different from one another.

When analyzing scores in a bell-shaped distribution, one might observe that two groups with high variability might have the same difference in means as two groups with low variability. However, we could conclude that in spite of the similarity in mean differences, the two groups with low variability appear more distinct or different from the groups with high variability, which overlap a great deal.

Viewing these bell curves helps us to understand that when looking for differences between scores for two groups, we must judge the difference between their means relative to the spread of the score. Researchers assume that good randomization in selecting or assigning events is in place before utilizing the t-test.

\section{Determining Statistical Significance}

Once researchers ascertain the $t$-value, they must look it up in a table of critical $t$-values to say whether the difference between the groups is likely to have been a chance finding. The risk level, which is called the alpha level, is usually set at .05 . This means that five times out of one hundred you could find a statistically significant difference between the means by chance.

Researchers must also determine the degrees of freedom (df), which is $n-2$. Given the alpha level, $t$-value, and df, researchers can use the critical value table to determine whether the $t$ value is large enough to be significant. If so, one can conclude that the difference between the means for the two groups is statistically significant, even given the variability (Reinard, 1998).

Statistical analysis plays a very important role in order to extract relevant phenomena for practical decisions. Two independent sample $t$-tests are for comparing means of two independent normally distributed populations. For large samples, the procedure often performs well even for nonnormal populations. The procedure will also produce confidence interval estimate for the difference of two means. The $t$-test is probably the most commonly used statistical test for this purpose. For example, you may wish to test the null hypothesis, $H_{0}: \mu_{1}-\mu_{2}=0$ at $\alpha$-significance level versus an alternative hypothesis such as

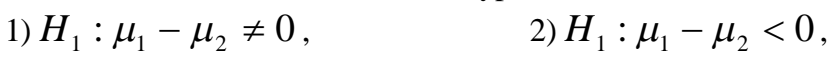
3) $H_{1}: \mu_{1}-\mu_{2}>0$. In general, the test is for comparing the mean of two sets of data, which can be expressed as:

$$
t=\frac{u_{1}-u_{2}}{s_{p} \sqrt{\frac{1}{n_{1}}+\frac{1}{n_{2}}}} \text { where, } t=\text { test statistic, } u_{1}=\text { mean of }
$$

first group, $u_{2}=$ mean of second group, $n_{1}=$ sample size of first group, $n_{2}=$ sample size of second group, $s_{p}=\frac{\left(n_{1}-1\right) s_{1}^{2}+\left(n_{2}-1\right) s_{2}^{2}}{n_{1}+n_{2}-2}$

with $S_{1}$ and $S_{2}$ being the standard deviation of group 1 and group 2, respectively (Hirsch \& Slack). One-sample $t$-test procedure can help you compare the mean (average) of a population with some hypothesized value, such as a business target. For example, you may wish to test the null hypothesis, $H_{0}: \mu=$ target, versus an alternative hypothesis such as $H_{1}: \mu \neq$ target. The one-sample $t$ statistic is: $t=\frac{\bar{x}-\mu_{0}}{\text { sem }}$

where, $\bar{x}$ represents the sample mean, $\mu_{0}$ represents the null value and 'sem' represents the estimated standard error of the mean calculated from the sample as: $\operatorname{sem}=\frac{s}{\sqrt{n}}$. This statistic has $n-1$ degrees of freedom.

The $t$-test was originally developed by the English statistician W.S. Gosset (1876-1937) while working for an Irish brewery. Gosset published under the pseudonym "Student" as secrecy was widely practiced in industrial circles (Plackett and Barnard, 1990).

Independent samples $t$-test and one sample $t$-test were used between comparison of the microbiological counts, physico- 
chemical values and standard values (PS: 1738 - 2000, Codex Stan 164-1989 and Gulf Standards, 2000).

\section{Result and Discussion}

\section{Discriminant Analysis (DA)}

Variation in fruit juice quality variables was evaluated through DA. The DA applied on raw data consisted of nine variables. Only one DF was found to discriminate the two types of fruit juices as shown in Table 1. Wilk's Lambda test showed that DF is statistically significant as shown in Table 2. Furthermore $100 \%$ of the total variance between the two types was explained by only one DF. The relative contribution for each variable is given in the following equation:

$Z=-17.00$ ash +13.86 protein +14.82 total sugar +17.89 reducing sugar+0.61 total soluble solid-0.81 arsenic +5.04 lead +12.68 zinc+27.01 copper

Table. 1. Wilks' Lambda for testing discriminant function validity

\begin{tabular}{|c|l|l|}
\hline Test of Function & Wilks' Lambda & P-value \\
\hline 1 & 0.001 & 0.000 \\
\hline
\end{tabular}

It can be seen that copper, reducing sugar, ash, total sugar, protein, zinc and lead exhibited strong contribution in discriminating the two types of fruit juice and account for most of the expected variations in juices quality, while other parameters showed less contribution in explaining the variation between Mango and Orange juices. The relative contribution for juices quality parameters can be arranged in the order.

Copper $>$ Reducing sugar $>$ Ash $>$ Total sugar $>$ Protein $>$ Zinc $>$ Lead $>$ Arsenic $>$ Total soluble solid.

Based on this ranking it may be concluded that copper, reducing sugar, ash, total sugar, protein, zinc and lead are the most important physical and chemical properties that discriminate mango and orange fruit juice. Total soluble solid is the least reliable physical parameter to discriminate the juice.

Table. 2. Classification results for discriminant analysis of the two types of fruit juices (a)

\begin{tabular}{|c|c|c|c|}
\hline Types & $\begin{array}{c}\% \\
\text { correct }\end{array}$ & \multicolumn{2}{|c|}{$\begin{array}{c}\text { Predicted Group } \\
\text { Membership }\end{array}$} \\
\cline { 3 - 4 } & & Orange & Mango \\
\hline Orange & 100.0 & 14 & 0 \\
Mango & 100.0 & 0 & 49 \\
\hline
\end{tabular}

(a) $100.0 \%$ of original grouped cases correctly classified.

The classification matrix (Table 2) showed that $100 \%$ of the cases were correctly classified to their respective groups. The results of classification also showed that significant differences existed between these two types of juice (Table 2), affording $100 \%$ correct assignation, which are expressed by in term of one discriminant function.

\section{Student's $\boldsymbol{t}$-tests}

Table 3 shows the result of the $t$-test. It is assumed that total sugar and reducing sugar content are increased with the advancement of ripening of fruits. Mango juices contained the higher $(\mathrm{p}<0.002)$ quantity of total sugar and reducing sugar.

The TSS content is significantly influenced by the combined effect of stages of maturity and ripening conditions. The TSS content of mango juices in this study was higher than orange juices $(\mathrm{p}=0.000)$ and for orange juices compare with standard value is statistically significant $(\mathrm{p}=0.0001)$ that means it is lower than the standard value. The variation of TSS of fruit juice depends on different source of juice types and fermentation of sugar caused by microorganisms.

Table. 3. $t$-test result for parameters in the analysis of fruit juices

\begin{tabular}{|l|c|c|}
\hline Parameters & $t$-value & p-value \\
\hline Total Sugar, \% & $-7.994^{\mathrm{a}}$ & 0.000 \\
\hline Reducing Sugar, \% & $-3.271^{\mathrm{a}}$ & \\
\hline Total Soluble Solid, \% & $-5.0018^{\mathrm{a}}$ & 0.0001 \\
& $11.61^{\mathrm{b}}$ & 0.0000 \\
\hline Acidity, \% & $-75.7051^{\mathrm{a}}$ & $>0.99999$ \\
\hline Ash, \% & $24.109^{\mathrm{a}}$ & $<0.00001$ \\
\hline Vitamin C (mg/100g) & $-94.4748^{\mathrm{a}}$ & $<0.00001$ \\
& $-1.924^{\mathrm{b}}$ & 0.029 \\
\hline Arsenic, mg/kg & $-442.2184^{\mathrm{a}}$ & $>0.99999$ \\
\hline Lead, mg/kg & $-35.5088^{\mathrm{a}}$ & $>0.99999$ \\
\hline Zinc, mg/kg & $-78.378^{\mathrm{a}}$ & $>0.99999$ \\
\hline Copper, mg/kg & $-82.386^{\mathrm{a}}$ & $>0.99999$ \\
\hline Standard plate count, & $-78.7401^{\mathrm{a}}$ & $>0.99999$ \\
cfu/ml & & \\
\hline
\end{tabular}

ane sample $t$-test and ${ }^{\mathrm{b}}$ independent samples $t$-test.

Citrus juices are a good option for gaining ascorbic acid (vitamin C). In this study mango juice might provide a level in excess vitamin $\mathrm{C}$ than orange juices $(\mathrm{p}=0.029)$. But the ascorbic acid content of commercial fruit juices is lost with respect to time and temperature during processing and storage. Many processors add ascorbic acid to their products to make up for processing losses. May be this is the cause for higher content of ascorbic acid in mango juices of the study.

\section{Conclusion and Policy Implications}

The multivariate statistical techniques, namely, discriminant analysis and $t$-test are important analytical techniques for the processing of fruit juices quality parameters and power full tools for classification as well as identification of possible factors of contamination. The techniques are also helpful in providing the possible mechanism with justification, by simple reasoning, to the causes of variation in fruit juices quality 
variables. This work has shown that the locally available fruit juices contain safe levels of nutritional and microbial elements and significant variation in nutritional information for human consumption. Each juice provides a different range of nutritional components that are desirable in a diet. The levels of all metals were within the limit of the BSTI standard for fruit juice. On the basis of standard plate count, about $100 \%$ of the samples recorded an acceptable range based on the Gulf standards for fruit juices. It was also found that the presence of pathogenic organisms such as total coliform, salmonella and fungus were within the acceptable range and considered safe for consumption. The government-authorized institute such as Bangladesh Council of Scientific and Industrial Research (BCSIR) and Bangladesh Standard and Testing Institute (BSTI) should undertake pre-emptive investigations to check the microbial and chemical quality of the fruit juices as well as initiate increase public awareness programs on contaminated and adulterated juices.

1. Codex Stan 164 (1989). General Standard for Fruit Juices, Joint FAO/WHO Food Standards Programme, US Codex Office, Food Safety and Inspection Service, US Department of Agriculture, Room 4861 South Building, 1400 Independence Ave., S.W., Washington, D.C. 202503700, USA.

2. Deanna M.M. \& S.B. Jeffrey (2007). Acid-alkaline balance: role in chronic disease and detoxification. Alternative Therapies 13(4): 62-65.

3. Doyle M.P., L.R. Beuchat \& T.J. Montville (2001). Food Microbiology. Washington DC: American Society for Microbiology, ASM Press.

4. Franke A.A., R.V. Cooney, S.M. Henning \& L.J. Custer (2005). Bioavailability and antioxidant effects of orange juice components in humans. J Agric Food Chem. 53 (13):5170-8.

5. Gulf Standards (2000). Microbiological Criteria for Foodstuffs, Part-1, GCC, Riyadh.

6. Horwitz W. (2003). Official Methods of Analysis of the Association of Official Analytical Chemists, $17^{\text {th }}$ ed. Washington DC, Association of Analytical Chemists.
7. James, C.S. (2004). Analytical Chemistry of Food. SealeHayne Faculty of Agriculture, Food and Land use, Department of Agriculture and Food Studies, University of Polymouth, UK, 1: 96-97.

8. Johnson R.A. \& D.W. Wichern (2007). Applied Multivariate Statistical Analysis. $6^{\text {th }}$ ed., Pearson Education.

9. Kurowska E.M., J.D. Spence, J. Jordan, S. Wetmore, D.J. Freeman, L.A. Piché \& P. HDL. Serratore (2000). Cholesterolraising effect of orange juice in subjects with hypercholesterolemia. Am J Clin Nutr 72:1095-100.

10. Lee J.Y., J.Y. Cheon, K.K. Lee, S.Y. Lee \& M.H. Lee (2001). Statistical evaluation of geochemical parameter distribution in a ground water system contaminated with petroleum hydrocarbons. Journal of Enviromental Quality 30, 1548-1563.

11. Mathew P.J. (1986). Agricultural and water management can there be peaceful co-existence. In Proceeding of $2^{\text {nd }}$ International Conference on Environmental Contamination, CEP Consultant, Armsterdam, 10-16

12. Plackett R.L., G.A. Barnard (1990). 'Student': A Statistical Biography of William Sealey Gosset: Based On Writings By E.S. Pearson. Oxford University Press.

13. PS: 1738 (2000). Pakistan Standard Specification for Orange Juice (2nd Rev.). Pakistan Standards and Quality Control Authority, Standards Development Centre, 39 - Garden Road, Saddar, Karachi-74400.

14. Saadia R., Tariq, H. Munir, N. Shah, A. Shaheen, S. Khalique, M. Manzoor, \& Jaffar (2005). Multivariate analysis of trace metal levels in tannery effluents in relation to soil and water: A case study from Peshawar, Pakistan. Journal of environmental management.

15. Srivastava R.P. \& K. Sanjeev (2003). Fruit and Vegetable Preservation Principles and Practices: Important Methods for Analysis of Fruits and Vegetables and their Products. $3^{\text {rd }}$ ed. International Book Distribution Co., Lucknow, India: 363p.

16. Tabachnick B.G. \& L.S. Fidell (1996). Using Multivariate Statistics. Harper Collins College Publishers: New York.

17. Underwood E.J. (1973). Trace elements. In Toxicant Occurring Foods. National Academy of Science, Washington, 43-87.

18. U.S. Food \& Drug Administration (FDA), CFSAN (2001) Bacteriological Analytical Manual Online. Chapter 12, pp 1-6. 
